\title{
repisälud
}

This is the peer reviewed version of the following article:

Hamczyk MR, Villa-Bellosta R, Andres V. In Vitro Macrophage Phagocytosis Assay. Methods Mol Biol. 2015;1339:235-46.

which has been published in final form at: https://doi.org/10.1007/978-1-4939-2929$\underline{016}$ 


\section{In vitro macrophage phagocytosis assay}

Magda R. Hamczyk, Ricardo Villa-Bellosta, Vicente Andrés

Laboratory of Molecular and Genetic Cardiovascular Pathophysiology, Vascular Biology Program, Centro Nacional de Investigaciones Cardiovasculares Carlos III (CNIC), Madrid, Spain

\section{Corresponding author:}

Vicente Andrés

CNIC

Telephone: +34-914531200, extension 1502

FAX: +34-91-4531265

Email: vandres@cnic.es

Running Head: Macrophage phagocytosis assay 


\section{Summary}

The key roles of macrophages in atherosclerosis include the phagocytosis of apoptotic and necrotic cells and cell debris, whose accumulation in atherosclerotic lesions exacerbates inflammation and promotes plaque vulnerability. Evidence is accumulating that macrophage phagocytic functions peak at early stages of atherosclerosis, and that the reduced phagocytosis at late stages of disease leads to the generation of necrotic cores and a defective resolution of inflammation, which in turn promotes plaque rupture, thrombus formation and life-threatening acute ischemic events (myocardial infarction and stroke). The impaired resolution of inflammation in advanced lesions featuring loss of macrophage phagocytic activity may be in part due to an imbalance between M1 and M2 subsets of polarized macrophages. A better understanding of the mechanisms that regulate macrophage phagocytic activity in the context of atherosclerosis may therefore help identify novel therapeutic targets. This chapter presents a protocol for establishing primary mouse macrophage cultures, a method for polarizing macrophages to the M1 and M2 states, and a method for the in vitro study of macrophage phagocytosis of IgG-opsonized or IgM/complementcomponent-3-opsonized erythrocytes.

Key Words. Phagocytosis, Macrophage, Opsonization, IgG, IgM, Complement Component 3, Red Blood Cells. 


\section{Introduction}

Atherosclerosis is the main feature of atherothrombotic vascular disease, the leading cause of death in developed countries which may soon acquire this status worldwide (1). Atherogenesis is initiated by the accumulation of lipoproteins in the subendothelial space of the arterial wall followed by the activation of endothelial cells $(2,3)$. Activated endothelium secrets chemokines that attract monocytes, which once within the lesion differentiate into macrophages upon exposure to macrophage colony-stimulating factor $(\mathrm{M}-\mathrm{CSF})(4,5)$. Once differentiated, lesional macrophages begin to ingest deposited lipoproteins through the scavenger receptor pathway (6). This process drives the transformation of macrophages into foam cells, which augment the inflammatory response and thus promote the recruitment of more monocytes and other inflammatory cells. Cytotoxic substances produced by foam cells cause the death of lesion-resident endothelial and smooth muscle cells $(7,8)$, producing enlarged lesions that contain dead cells, cell debris, lipids and extracellular matrix. A major function of macrophages is to ingest these components through phagocytosis. Macrophage phagocytic activity is high in the initial stages of atherosclerosis, but in advanced plaques it is defective and macrophages fail to engulf dead cells and cell debris, compromising the resolution of inflammation. Necrotic cores then accumulate and generate life-threatening vulnerable plaques that can easily undergo rupture, causing thrombus formation and myocardial infarction or stroke.

Phagocytosis is a receptor-mediated process by which cells ingest large particles $(\geq$ $0.5 \mu \mathrm{m})$. Since macrophages can encounter a great variety of particles, they express many receptors that can mediate phagocytosis. Moreover, some molecules, such as antibodies and complement components (e.g. complement component 3, C3), 
facilitate phagocytosis by tagging particles for recognition, in a process called opsonization. The two best-studied phagocytic receptors are Fc $\gamma$ receptor $(\mathrm{Fc} \gamma \mathrm{R})$ and $\mathrm{C} 3$ receptor (CR3), which recognize $\mathrm{IgG}$ and $\mathrm{C} 3$-opsonized particles, respectively. In this chapter we describe a protocol for the in vitro study of these two phagocytotic routes using macrophage primary cultures. Because an imbalance between proinflammatory M1 and anti-inflammatory M2 macrophages in large lesions is thought to contribute to impaired resolution of inflammation (9), we describe an optional step for the polarization of macrophages to the M1 (classical) and M2 (alternative activation) phenotypes. The workflow for the complete protocol is shown in Figure 1.

[Figure 1 near here] 


\section{Materials}

\subsection{Establishing macrophage primary culture}

1. Mice.

2. $70 \%$ ethanol in distilled $\mathrm{H}_{2} \mathrm{O}$.

3. Dissection material: tweezers, scissors and sterile scalpel blades.

4. Conical sterile polypropylene centrifuge tubes $(15 \mathrm{~mL})$.

5. Phosphate buffered saline (PBS): $1.54 \mathrm{mM} \mathrm{KH}_{2} \mathrm{PO}_{4}, 155.17 \mathrm{mM} \mathrm{NaCl}$ and 2.71 $\mathrm{mM} \mathrm{Na} 2 \mathrm{HPO}_{4}, \mathrm{pH} 7.4$, sterilized by autoclaving.

6. Roswell Park Memorial Institute (RPMI) 1640 medium with antibiotics: 100 units/mL penicillin and $100 \mu \mathrm{g} / \mathrm{mL}$ streptomycin (see Note $\mathbf{1}$ ).

7. Cell culture dishes $(60 \mathrm{~mm}$ and $100 \mathrm{~mm})$.

8. Paper towels.

9. Syringes $(10 \mathrm{~mL})$ and needles $(25 \mathrm{G})$.

10. Distilled $\mathrm{H}_{2} \mathrm{O}$.

11. Heat-inactivated $\left(30\right.$ minutes at $\left.56^{\circ} \mathrm{C}\right)$ fetal bovine serum (FBS).

12. Differentiation medium: RPMI 1640 supplemented with $10 \%$ heat-inactivated FBS, 20\% L-cell conditioned medium (see Note 2), 2 mM L-glutamine, 100 units $/ \mathrm{mL}$ penicillin, and $100 \mu \mathrm{g} / \mathrm{mL}$ streptomycin.

\subsection{Seeding macrophages on coverslips}

1. 24-well cell culture plates.

2. Round coverslips (matching the well size, e.g. $12 \mathrm{~mm}$ for 24 -well plates).

3. PBS (as in section 2.1., item 5).

4. Differentiation medium (as in section 2.1., item 12).

5. Sterile cell scrapers. 
6. Conical sterile polypropylene centrifuge tubes $(50 \mathrm{~mL})$.

7. Cell counting chamber.

\subsection{Macrophage polarization (optional)}

1. M1 polarization medium: RPMI 1640 supplemented with $10 \%$ heat-inactivated FBS (see section 2.1., item 11), 2 mM L-glutamine, 100 units/mL penicillin, 100 $\mu \mathrm{g} / \mathrm{mL}$ streptomycin, $100 \mathrm{ng} / \mathrm{mL}$ lipopolysaccharide (LPS), and $10 \mathrm{ng} / \mathrm{mL}$ interferon $\gamma$ (INF $\gamma)$

2. M2 polarization medium: RPMI 1640 supplemented with $10 \%$ heat-inactivated FBS (see section 2.1., item 11), 2 mM L-glutamine, 100 units/mL penicillin, 100 $\mu \mathrm{g} / \mathrm{mL}$ streptomycin, and $10 \mathrm{ng} / \mathrm{mL}$ interleukin 4 (IL-4).

3. Differentiation medium (as in section 2.1., item 12).

4. PBS (see section 2.1., item 5).

5. Permanent marker

\subsection{Phagocytosis assay}

1. PBS (see section 2.1., item 5).

2. Starvation medium: RPMI 1640 medium containing $0.1 \%$ heat-inactivated FBS (as in section 2.1., item 11), $2 \mathrm{mM}$ L-glutamine, 100 units $/ \mathrm{mL}$ penicillin, and $100 \mu \mathrm{g} / \mathrm{mL}$ streptomycin.

3. Sheep red blood cells (RBCs) in Alsever's solution (see Note 3) (e.g., Cat. No. SR0053, Oxoid).

4. Polypropylene microcentrifuge tubes $(1.5 \mathrm{~mL})$.

5. $20 \mathrm{mM}$ glucose in HBSS (see Note 4). 
6. Rabbit IgM fraction anti-sheep RBCs (e.g., Cat. No. CL9000-M, Cedarlane) (see Note 5).

7. Rabbit IgG fraction anti-sheep RBCs (e.g., Cat. No. 55806, Cappel) (see Note 5).

8. Complement component 5 (C5)-deficient serum (e.g., Cat. No. C1163, Sigma) (see Note 6).

9. Conical sterile polypropylene centrifuge tubes $(50 \mathrm{~mL})$.

10. Ice.

\subsection{Cell fixing and immunostaining}

1. PBS (as in section 2.1., item 5).

2. $4 \%$ formaldehyde in PBS (for $500 \mathrm{~mL}$ : in a fume hood heat $400 \mathrm{~mL}$ of PBS to aprox. $60^{\circ} \mathrm{C}$ on a hot plate with magnetic stirrer, add $20 \mathrm{~g}$ of paraformaldehyde and incubate with mixing, add dropwise $1 \mathrm{M} \mathrm{NaOH}$ until all powder is dissolved, fill up with PBS up to $500 \mathrm{~mL}$, cool the solution, filter and adjust $\mathrm{pH}$ to 7.4, use fresh or freeze at $\left.-20^{\circ} \mathrm{C}\right)$.

3. $0.5 \%(\mathrm{v} / \mathrm{v})$ Triton $\mathrm{X}-100$ in PBS.

4. $0.1 \%(\mathrm{v} / \mathrm{v})$ Triton $\mathrm{X}-100$ in PBS.

5. Blocking solution: PBS supplemented with $1 \%(\mathrm{w} / \mathrm{v})$ bovine serum albumin (BSA) and $0.1 \%(\mathrm{v} / \mathrm{v})$ Triton $\mathrm{X}-100$.

6. Anti-rabbit secondary antibody conjugated with a fluorochrome of choice (e.g. FITC, AlexaFluor488, etc.).

7. Mounting medium of choice containing diamidino-2-phenylindole (DAPI; e.g. ProLong Gold Antifade Mountant with DAPI, Molecular Probes, see Note 7).

8. Microscope glass slides (e.g. 76 x $26 \mathrm{~mm}$ ).

9. Fluorescence or confocal microscope. 


\section{Methods}

\subsection{Establishing macrophage primary culture}

1. Euthanize a mouse, from which the lower limbs will be obtained (see Note 8).

2. Spray the mouse with $70 \%$ ethanol to disinfect.

3. Using tweezers and scissors remove the skin from both legs (see Note 9).

4. Cut away each leg at the hip joint (where the head of the femur meets the pelvis), and place them in a $15 \mathrm{~mL}$ tube containing PBS.

5. In a flow cabinet, transfer the legs to a paper towel (see Note 10) and remove muscles with a sterile blade to obtain clean femurs and tibias.

6. Put the bones on a $60 \mathrm{~mm}$ dish containing RPMI medium with antibiotics.

7. Hold one bone with sterile tweezers and cut both joints with sterile scissors to gain access to the bone marrow (see Note 11).

8. Aspirate RPMI medium with antibiotics into a $10 \mathrm{~mL}$ syringe and fit a $25 \mathrm{G}$ needle.

9. Introduce the needle into the bone lumen and flush out the bone marrow into a new $60 \mathrm{~mm}$ dish until the bone color turns white.

10. Aspirate the medium containing the bone marrow using the same syringe and pass through the needle to disaggregate cells.

11. Repeat steps $7-10$ for all the bones.

12. Transfer the cell suspension from the dish to a $15 \mathrm{~mL}$ tube and centrifuge at $300 x$ $g$ for 5 minutes at room temperature.

13. Discard the supernatant and add $1 \mathrm{~mL}$ of distilled $\mathrm{H}_{2} \mathrm{O}$ to lyse RBCs. Pipette up and down for 10 seconds and add PBS to $15 \mathrm{~mL}$.

14. Centrifuge at $300 \times g$ for 5 minutes at room temperature. Repeat the lysis step if after centrifugation the pellet still contains RBCs. 
15. Discard the supernatant and resuspend the pellet in $45 \mathrm{~mL}$ differentiation medium (see Note 12).

16. Plate the cells on four $100 \mathrm{~mm}$ dishes ( $10 \mathrm{~mL}$ of cell suspension per dish).

17. Incubate at $37^{\circ} \mathrm{C}$ in a $5 \% \mathrm{CO}_{2}$ atmosphere.

18. After $48 \mathrm{~h}$ (day 2) of culture add $10 \mathrm{~mL}$ of fresh differentiation medium (see Note

13) to each plate. At this point some attached cells can be observed (see Figure 2, top).

19. After further $48 \mathrm{~h}$ (day 4 ) of culture, discard the medium and add $10 \mathrm{~mL}$ of fresh differentiation medium to each plate.

20. After a further $48 \mathrm{~h}$ (day 6 ) of culture, discard the medium and add $10 \mathrm{~mL}$ of fresh differentiation medium to each plate. At this point the attached cells usually reach confluence (see Figure 2, bottom).

21. After 7 days in culture, bone marrow procursors are considered fully differentiated to macrophages (M0 state) and are ready for experiments.

[Figure 2 near here]

\subsection{Seeding macrophages on coverslips}

1. In the flow cabinet, prepare a 24-well plate (see Note 14).

2. Put a glass coverslip in each well and expose the plate to UV light for 20 minutes in order to sterilize it.

3. Aspirate medium from one $100 \mathrm{~mm}$ dish containing a confluent monolayer of primary macrophages (see Note 15) and wash twice with PBS to remove dead and unattached cells. 
4. Add fresh differentiation medium and gently scrape off the macrophages with a cell scraper (see Note 16).

5. Transfer the medium containing cells to a $50 \mathrm{~mL}$ tube and centrifuge at $260 \times \mathrm{g}$ for 5 minutes at room temperature.

6. Discard the supernatant and resuspend the cell pellet in differentiation medium.

7. Count the cells in a cell counting chamber (see Note 17).

8. Prepare a suspension containing $5 \times 10^{4}$ cells per $\mathrm{mL}$ of differentiation medium (e.g. for one 24-well plate prepare around $1.25 \times 10^{6}$ cells in $25 \mathrm{~mL}$ ).

9. Transfer $1 \mathrm{~mL}$ of the cell suspension (around $5 \times 10^{4}$ cells, see Note 18) into each well containing a coverslip (prepared in step 2) and briefly shake plates to distribute the cells evenly.

10. Incubate cells at $37^{\circ} \mathrm{C}$ in a $5 \% \mathrm{CO}_{2}$ atmosphere for a few hours (or overnight) to allow cells to attach.

11. If phagocytic activity is to be examined in polarized macrophages, proceed as indicated in section 3.3. For phagocytic assays with M0 macrophages, go directly to section 3.4.

\subsection{Macrophage polarization (optional)}

1. Prepare polarization medium for macrophages $(1 \mathrm{~mL}$ for each well of a 24 well plate) just before use (see Note 19).

2. Remove medium from all wells (from step 10 in the previous section) and wash them once with PBS.

3. Add the appropriate polarization (M1 and M2) or differentiation (M0) medium to each well. Identify wells on the plate cover using a marker.

4. Incubate at $37^{\circ} \mathrm{C}$ in a $5 \% \mathrm{CO}_{2}$ atmosphere for $16-24 \mathrm{~h}$. 


\subsection{Phagocytosis assay}

1. Wash the macrophages once with PBS and incubate with starvation medium for 2 hours (see Note 20) at $37^{\circ} \mathrm{C}$ in a $5 \% \mathrm{CO}_{2}$ atmosphere.

2. Mix sheep RBC solution by flipping the tube, and transfer an appropriate volume (depending on the design of your experiment) to two $1.5 \mathrm{~mL}$ tubes, one for $\operatorname{IgM}$ opsonization and another for IgG opsonization. Use $0.5 \mu \mathrm{L} \mathrm{RBC}$ solution for each well of a 24-well plate. Remember to leave some control wells free of RBCs.

3. Wash RBCs by adding $1 \mathrm{~mL}$ of $20 \mathrm{mM}$ glucose in HBSS, centrifuge at $1500 \times \mathrm{g}$ for 4 minutes at room temperature, and discard the supernatant.

4. Repeat step 3 (see Note 21).

5. Resuspend each RBC pellet in $0.25 \mathrm{~mL}$ of $20 \mathrm{mM}$ glucose in HBSS.

6. Prepare the IgM and IgG solutions: add $3 \mu \mathrm{L}$ of anti-sheep RBC IgM or $0.1 \mu \mathrm{L}$ of anti-sheep RBC IgG per $\mu \mathrm{L}$ of RBC solution used in step 2 to $0.25 \mathrm{~mL}$ of $20 \mathrm{mM}$ glucose in HBSS.

7. Mix RBC solutions (obtained in step 5) with $\operatorname{IgG}$ or IgM solution (obtained in step $6)$.

8. Incubate for 30-60 minutes at room temperature with gentle mixing to enable opsonization (see Note 22).

9. Centrifuge at $1500 \times g$ for 4 minutes at room temperature, discard the supernatant and resuspend each pellet in $1 \mathrm{~mL}$ of $20 \mathrm{mM}$ glucose in HBSS (see Note 23).

10. Centrifuge at $1500 \times g$ for 4 minutes at room temperature, and discard the supernatant. Store the pellet of IgG-opsonized RBCs at $4{ }^{\circ} \mathrm{C}$ while performing steps 11-13 with the IgM-opsonized RBCs. 
11. Mix $20 \mu \mathrm{L}$ of C5-deficient serum (containing C3) with $180 \mu \mathrm{L}$ of $20 \mathrm{mM}$ glucose in HBSS to obtain $200 \mu \mathrm{L}$ of diluted C5-deficient serum.

12. Resuspend IgM-opsonized RBC pellet in $200 \mu \mathrm{L}$ of diluted C5-deficient serum (containing C3) and incubate at $37^{\circ} \mathrm{C}$ for 30 minutes with mixing.

13. Add $1 \mathrm{~mL}$ of $20 \mathrm{mM}$ glucose in HBSS to the same tube (IgM/RBCs sample), centrifuge at $1500 \times \mathrm{g}$ for 4 minutes at room temperature, and discard the supernatant. 14. Resuspend in $50 \mathrm{~mL}$ tubes the complexes containing $\mathrm{RBC} / \mathrm{IgG}$ (obtained in step 10) or $\mathrm{RBC} / \mathrm{IgM} / \mathrm{C} 3$ (obtained in step 13), using an appropriate volume of starvation medium (calculate $1 \mathrm{~mL}$ of starvation medium for each well).

15. Aspirate the starvation medium from plates containing cultured macrophages (prepared in section 3.4, step 1) and add $1 \mathrm{~mL}$ per well of medium containing $\mathrm{RBC} / \mathrm{IgG}$ or $\mathrm{RBC} / \mathrm{IgM} / \mathrm{C} 3$ complexes (see Note 24 ). Incubate 15 minutes at $37^{\circ} \mathrm{C}$ in $5 \% \mathrm{CO}_{2}$ atmosphere. Remember to leave some wells without RBCs.

16. Place the plates on ice and wash wells $2-3$ times with $1-2 \mathrm{~mL}$ PBS to arrest the phagocytosis process. Proceed directly to step 1 in section 3.5 .

\subsection{Cell fixing and immunostaining}

1. Fix the macrophages (from step 16 in section 3.4) by incubating with $4 \%$ formaldehyde (in a fume hood) for 10-15 minutes at room temperature (see Note 25). 2. Rinse twice with PBS (see Note 26).

3. Permeabilize the cells with $0.5 \%$ Triton $\mathrm{X}-100$ in PBS for 10 minutes at room temperature.

4. Wash three times with $0.1 \%$ Triton X-100 in PBS for 5 minutes.

5. To block non-specific binding of the antibodies, incubate coverslips with blocking solution for 30 minutes at room temperature. 
6. Prepare a dilution of the antibody conjugated to a fluorochrome in blocking solution; allow around 150-200 $\mu$ l per coverslip (see Note 27).

7. Remove the blocking solution by holding each coverslip on its edge with forceps and touching a paper towel.

8. Immediately incubate the coverslips with diluted antibody for 1 hour at room temperature in a humidified chamber in the dark.

9. Aspirate the antibody solution and wash coverslips 3 times with $0.1 \%$ Triton $\mathrm{X}-100$ in PBS, then once with PBS (5 minutes each wash).

10. Using tweezers, take each coverslip and remove liquid by touching the corner of a paper towel. Invert the coverslip on a drop of mounting medium (with DAPI) placed on a glass slide. Mount 2-3 coverslips per glass slide.

11. Allow the slide to dry in the dark over night at $4{ }^{\circ} \mathrm{C}$ or proceed directly to the next step, depending on the mounting medium used (see supplier instructions).

12. Under a fluorescence or confocal microscope, count the number of phagocytized RBCs in individual macrophages.

13. Calculate the phagocytic index, which is usually given as the average number of erythrocytes per 100 macrophages. You can also determine the percentage of macrophages that engulfed at least one erythrocyte. 


\section{Notes}

1. Medium can also contain L-glutamine and heat-inactivated fetal bovine serum (medium composition is not crucial at this point). Instead of medium, sterile PBS can be used.

2. L-cell conditioned medium (LCM) is a source of macrophage colony-stimulating factor (M-CSF) which drives differentiation of progenitor cells to macrophages. Differentiation medium can be supplemented with $100 \mathrm{U} / \mathrm{mL}$ recombinant M-CSF instead of $20 \%$ LCM. This increases the cost of the experiment but gives more reliable and reproducible results.

3. Alsever's solution is an isotonic, balanced salt solution widely used as a blood preservative, permitting prolonged storage under refrigeration and preserving the antigenic properties of erythrocytes. Sheep RBCs in Alsever's solution are available normally in $20 \mathrm{~mL}$ vials and have quite short expiry date (around 2-3 months), so before each experiment make sure that the reagent has not expired.

4. Alternatively, glucose can be dissolved in PBS.

5. Many suppliers do not give the exact concentrations of the $\operatorname{IgG}$ and $\operatorname{IgM}$ fractions, so the amount of antibodies used for opsonization normally requires optimization.

6. Complement $\mathrm{C} 5$-deficient serum contains $\mathrm{C} 3$, which binds to $\operatorname{IgM}$ antibodies on the RBC surface and can then be recognized by CR3 (also called Mac-1) on macrophages. Serum used for the experiment should lack C5 to avoid activation of the alternative complement pathway, which would result in erythrocyte lysis! 7. It is also possible to use mounting medium without nuclear stain and perform an additional staining step (after step 9 in section 3.5) with DAPI or Hoechst. 8. The protocol description is for one mouse. Adjust amounts and volumes to the number of mice required for your experiment. 
9. Femurs and tibias are the richest source of murine bone marrow. If the number of mice is limited, extract additional marrow from the upper limbs.

10. To minimize the risk of contamination you can incubate the legs in $70 \%$ ethanol for 1 minute and wash once with PBS just before removing the muscles.

11. In case of tibia it is necessary to cut away almost half of the bone (from the side where the foot and the leg meet). To avoid contamination after opening a bone, do not put it down until the bone marrow is flushed out.

12. Bone marrow progenitors have a high proliferation potential and normally one animal is enough to yield sufficient macrophages for a phagocytosis assay. For all experiments maintain similar seeding density, but if counting cells bear in mind that bone marrow consists of different progenitors, and macrophage precursors are just a fraction of this. We typically resuspend the bone marrow in $45 \mathrm{~mL}$ medium to seed on four $100 \mathrm{~mm}$ dishes, but the volume can differ depending on the experiment.

13. At this point it is better to add medium (instead of changing it) because there are still many unattached macrophage progenitors.

14. Although the protocol is given for 24-well plates, it can be adjusted for other formats. In any case, remember to dedicate some wells as technical controls for immunostaining, e.g. do not add RBCs to some wells to have negative controls (wells where no fluorescence should be detected). These wells will be a reference of unspecific fluorescence or background.

15. Instead of primary cell cultures, some established macrophage cell lines can be used, such as RAW264.7; however, the phagocytosis period needs to be extended, usually up to 30 minutes. The phagocytic activity of some cell lines might need to be augmented by stimulation with phorbol myristate acetate (PMA). 
16. Alternatively, wash the cells twice with PBS and detach them with $2 x$ or $4 x$ trypsin. Bear in mind that macrophages are strongly attached to the plate surface so do not use $1 \mathrm{x}$ trypsin as it may be ineffective.

17. A Neubauer chamber can be used, or any other cell counting chamber available in your laboratory.

18. The number of macrophages seeded on each well can vary from $2.5 \times 10^{4}$ to $1.5 \mathrm{x}$ $10^{5}$ cells and may require some optimization depending on the cell type and mouse genotype. Seeding densities that are too high or too low can make it difficult to quantify RBC-to-macrophage ratios. If cells are too close to each other, it is difficult to assess which of the adjacent cells engulfed a particular erythrocyte. On the other hand, if cells are seeded too sparsely many photographs are needed to obtain sufficient numbers of cells for RBC/macrophage quantification.

19. Macrophage polarization is an optional step that depends on the experimental strategy. As an alternative, other stimuli or drugs can be applied before the phagocytosis assay.

20. It is also possible to starve macrophages in medium without FBS. The duration of starvation may be extended but the same duration must be maintained for all experiments.

21. The purpose of these washes is to eliminate broken cells from the RBC stock solution before using it. At the end of step 4 the supernatant should be free of signs of hemolysis. Two washes are normally enough.

22. If available, use a noria mixer as it will provide the best conditions for opsonization. 
23. It normally takes a while to resuspend the pellet, but this is a sign the opsinization was successful.

24. At this point, it is possible to add a short centrifugation step (300 $x \mathrm{~g}$ for 1 minute at room temperature) to synchronize the phagocytosis process.

25. In order to save time, perform steps 1-5 (section 3.5) directly on the 24-well plate containig coverslips (since volumes of the fixation, permeabilization and blocking solutions do not have to be exact).

26. Plates can be stored at $4{ }^{\circ} \mathrm{C}$ for quite a long time before processing. It is important to fill up all the wells with PBS and wrap each plate with parafilm to avoid evaporation.

27. The antibody dilution may vary depending on the source, and normally needs to be optimized. Remember to use an antibody that recognizes antibodies involved in the opsonization process, e.g. if rabbit IgGs and IgMs were used to form complexes with RBCs, for the detection use a secondary antibody anti-rabbit $\operatorname{IgG/IgM}$ made in a different species, e.g. donkey or goat. 


\section{Acknowledgements}

We thank Simon Bartlett for English editing. M.R.H. is supported by a FPI predoctoral fellowship from the Spanish Government (BES-2011-043938) and R.VB. by a Juan de la Cierva postdoctoral contract from the Spanish Government (JCI2011-09663). Work in V.A.'s laboratory is supported by grants from the Spanish Ministry of Economy and Competitivity (MINECO) (SAF2013-46663-R), Fondo Europeo de Desarrollo Regional (FEDER), Instituto de Salud Carlos III (RD12/0042/0028), The Progeria Research Foundation (Innovator Award 2012, Established Investigator Award 2014), and the European Union (Liphos, Grant Agreement 317916). The Centro Nacional de Investigaciones Cardiovasculares (CNIC) is supported by the MINECO and the Pro-CNIC Foundation. 


\section{References}

1. Lloyd-Jones D, Adams RJ, Brown TM et al. (2010) Executive summary: heart disease and stroke statistics - 2010 update: a report from the American Heart Association. Circulation 121(7):948-54

2. Williams KJ, Tabas I (1995) The response-to-retention hypothesis of early atherogenesis. Arterioscler Thromb Vasc Biol 15(5):551-61

3. Tabas I, Williams KJ, Boren J (2007) Subendothelial lipoprotein retention as the initiating process in atherosclerosis: update and therapeutic implications. Circulation 116(16):1832-44

4. Glass CK, Witztum JL (2001) Atherosclerosis. the road ahead. Cell 104(4):503-16 5. Mestas J, Ley K (2008) Monocyte-endothelial cell interactions in the development of atherosclerosis. Trends Cardiovasc Med 18(6):228-32

6. Greaves DR, Gordon S (2005) Thematic review series: the immune system and atherogenesis. Recent insights into the biology of macrophage scavenger receptors. $J$ Lipid Res 46(1):11-20

7. Kockx MM, Knaapen MW (2000) The role of apoptosis in vascular disease. $J$ Pathol 190(3):267-80

8. Boyle JJ, Weissberg PL, Bennett MR (2002) Human macrophage-induced vascular smooth muscle cell apoptosis requires NO enhancement of Fas/Fas-L interactions. Arterioscler Thromb Vasc Biol 22:1624-1630

9. Johnson JL, Newby AC (2009) Macrophage heterogeneity in atherosclerotic plaques. Curr Opin Lipidol 20(5): 370-378 


\section{FIGURE LEGENDS}

Figure 1. Work flow of the phagocytosis assay with estimated times for each step. MФ, macrophages; RBCs, red blood cells; C3, complement component 3.

Figure 2. Primary macrophage cell culture. Mouse bone marrow progenitors were cultured in RPMI 1640 medium containing 10\% heat-inactivated fetal bovine serum, 20\% L-cell conditioned medium, 2 mM L-glutamine, 100 units/mL penicillin, and $100 \mu \mathrm{g} / \mathrm{mL}$ streptomycin. Pictures show representative cell cultures after 2 days (top) and 6 days (bottom). 

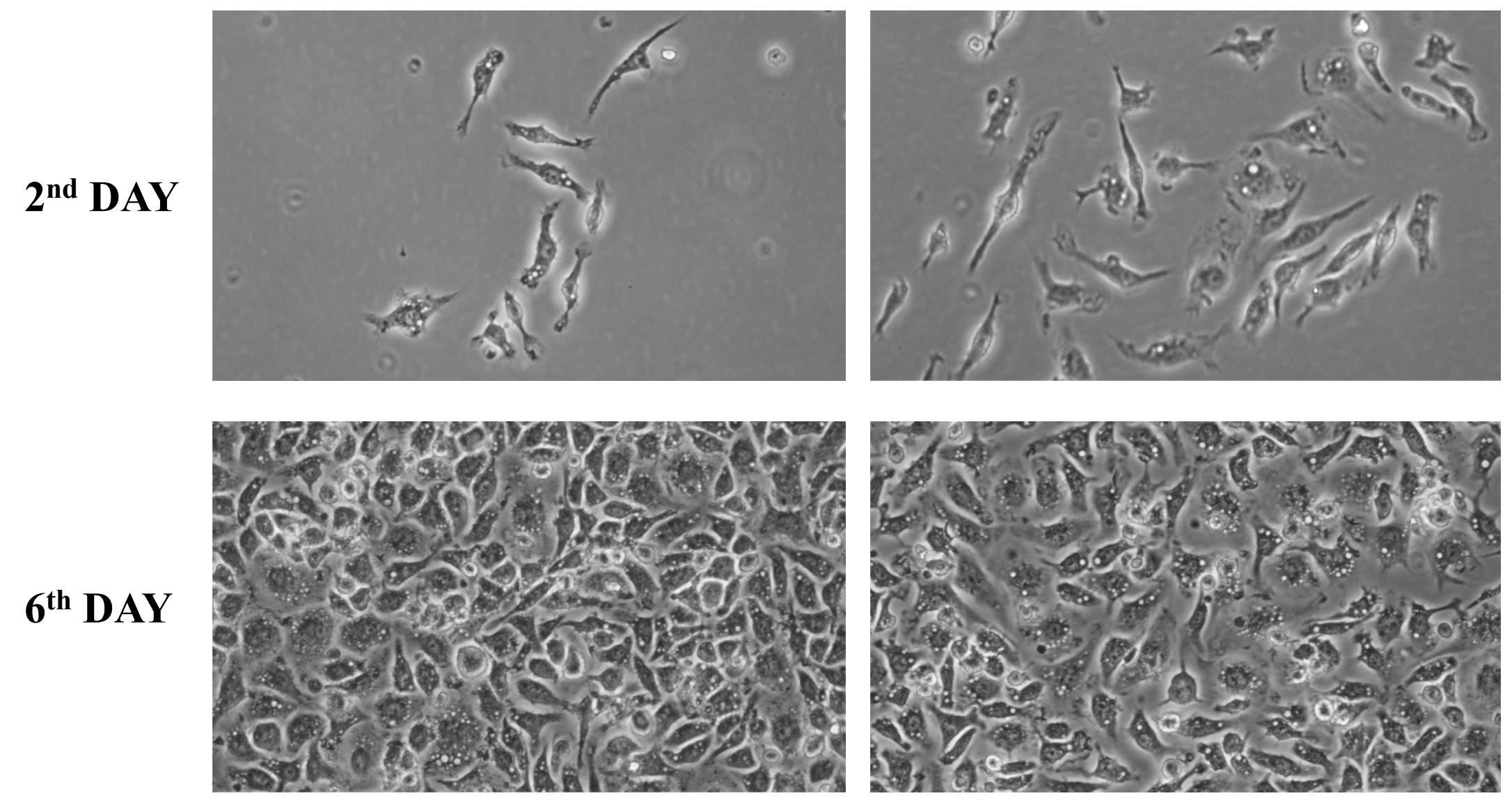